\title{
Article \\ Rethinking Carbohydrate Intake and Time in Range in Children and Adolescents with Type 1 Diabetes
}

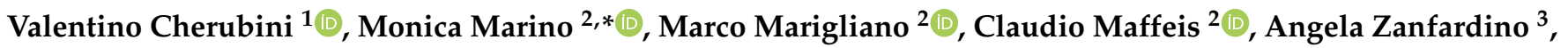 \\ Ivana Rabbone ${ }^{4}$, Sara Giorda ${ }^{5}$, Riccardo Schiaffini ${ }^{6}$, Antonella Lorubbio ${ }^{6}$, Serena Rollato ${ }^{3}$, Antonio Iannilli ${ }^{1}$, \\ Dario Iafusco $^{3}$, Andrea E. Scaramuzza ${ }^{7}$ (), Renee Bowers ${ }^{8}$ and Rosaria Gesuita ${ }^{9}$
}

check for updates

Citation: Cherubini, V.; Marino, M.; Marigliano, M.; Maffeis, C.;

Zanfardino, A.; Rabbone, I.; Giorda,

S.; Schiaffini, R.; Lorubbio, A.; Rollato, S.; et al. Rethinking Carbohydrate Intake and Time in Range in Children and Adolescents with Type 1 Diabetes. Nutrients 2021, 13, 3869. https://doi.org/10.3390/nu13113869

Academic Editor: Roberto Cangemi

Received: 5 October 2021

Accepted: 28 October 2021

Published: 29 October 2021

Publisher's Note: MDPI stays neutral with regard to jurisdictional claims in published maps and institutional affiliations.

Copyright: (C) 2021 by the authors. Licensee MDPI, Basel, Switzerland. This article is an open access article distributed under the terms and conditions of the Creative Commons Attribution (CC BY) license (https:/ / creativecommons.org/licenses/by/ $4.0 /)$.
1 Department of Women's and Children's Health, G. Salesi Hospital, 60123 Ancona, Italy; valentino.cherubini@gmail.com (V.C.); Antonio.Iannilli@ospedaliriuniti.marche.it (A.I.)

2 Pediatric Diabetes and Metabolic Disorders Unit, University of Verona, 37100 Verona, Italy; marco.marigliano@univr.it (M.M.); claudio.maffeis@univr.it (C.M.)

3 Regional Center of Pediatric Diabetology, University of Campania "L. Vanvitelli", 80100 Naples, Italy; angela.zanfardino@unicampania.it (A.Z.); serenarollato@gmail.com (S.R.); dario.iafusco@unicampania.it (D.I.)

4 Department of Health and Science, University of Oriental Piedmont, 28100 Novara, Italy; ivana.rabone@uniupo.it

5 Department of Medical Sciences, University of Turin, 10092 Turin, Italy; saragiorda.91@gmail.com

6 Diabetes Unit, Pediatric Hospital Bambino Gesù, 00031 Roma, Italy; riccardo.schiaffini@gmail.com (R.S.); antonella.lorubbio@opbg.net (A.L.)

7 Division of Pediatrics, Pediatric Diabetes, Endocrinology and Nutrition, ASST Cremona, 26100 Cremona, Italy; a.scaramuzza@gmail.com

8 Population Health, Faculty of Health Sciences, University of Ottawa, 64 Sherbrooke Avenue, Ottawa, ON 61350, Canada; renee.bowers13@gmail.com

9 Center of Epidemiology and Biostatistics, Polytechnic University of Marche, 60123 Ancona, Italy; r.gesuita@staff.univpm.it

* Correspondence: monicamarino96@gmail.com

Abstract: The aim of this study was to evaluate the association between macronutrient intake and time in range (TIR) of 70-180 mg/dL in children and adolescents with type 1 diabetes (T1D). A multi-center study recruited patients with T1D using continuous glucose monitoring (CGM) between January 2019 and January 2020 from centers across Italy. Diet intake was recorded using three-day weighed food diaries. Nutrients were evaluated as percentages of total intake. TIR was considered at target if the percentage of readings was higher than $70 \%$. Clinical and nutritional factors associated with TIR at target were analyzed using multiple correspondence analysis and multiple logistic regression. Data from 197 participants (53\% male, median age 11.6 years, median HbA1c $55.2 \mathrm{mmol} / \mathrm{mol}$, median TIR $60 \%$ ) were analyzed. Macronutrient intake was $45.9 \%$ carbohydrates, $16.9 \%$ protein, $37.3 \%$ fat, and $13.1 \mathrm{~g} /$ day fiber (median values). TIR $>70 \%$ was observed in $28 \%$ of participants; their diet contained more protein $(17.6 \%, p=0.015)$ and fiber $(14.4 \mathrm{~g} /$ day, $p=0.031)$ than those not at target. The probability of having a TIR $>70 \%$ was significantly higher with $40-44 \%$ consumption of carbohydrates compared with $45-50 \%$ consumption of carbohydrates and with the use of a carbohydrate counting system. Based on these results, a five percent reduction in the percentage of carbohydrate intake can help children and adolescents with T1D achieve the goal of a TIR $>70 \%$. Both a lower and higher percentage of carbohydrate intake appears to reduce the probability of reaching the target TIR $>70 \%$. These results require validation in other populations before being used in clinical practice.

Keywords: time in range; macronutrients; children and adolescents; type 1 diabetes; multi-center observational study; continuous glucose monitoring 


\section{Introduction}

Medical nutrition therapy is a cornerstone of diabetes management and includes education on carbohydrate estimation as part of an overall eating pattern to improve health outcomes [1]. Monitoring carbohydrate intake, whether by carbohydrate counting or experience-based estimation, remains a key strategy in achieving glycemic control [2]. Nevertheless, it has been shown that the dietary intake of young people with type 1 diabetes (T1D) in the community generally fails to meet recommended nutrient intakes as outlined in the International Society for Pediatric and Adolescent Diabetes (ISPAD) dietary guidelines for T1D [3,4]. In a large cohort of young people with T1D, the overall intake of total and saturated fats was high, while intake of fruits, vegetables, and grains was low [5]. Cross-sectional studies on children with T1D have suggested that diets characterized by lower fat [6], lower added sugar [7], higher carbohydrates [8], higher fiber, and higher fruit and vegetable consumption [6-8] are associated with lower glycated hemoglobin $(\mathrm{HbA} 1 \mathrm{c})$. Nutrition analysis in patients with T1D is relevant, because both overall diet quality and macronutrient distribution are associated with improved glycemic control [9]. Measurement of $\mathrm{HbA} 1 \mathrm{c}$ is currently the most important method used to evaluate glycemic control, but continuous glucose monitor (CGM)-based percentage of time spent in the target range $70-180 \mathrm{mg} / \mathrm{dL}$ (TIR) has been considered an emerging measure of glycemic control because it is positively associated with reduced micro and macrovascular complications [10,11]. The use of both non-automated [12] and automated diabetes technologies such as closed loop control systems (CLC) have been shown to improve TIR [13]. The aim of this study was to analyze the association between clinical factors and macronutrient intake with CGM-based TIR in children and adolescents aged 2-17 years in the real-world community setting.

\section{Materials and Methods}

Pediatric diabetes centers with experience in the use of CGM and the availability of a specialized pediatric dietitian in childhood diabetes were invited to participate in this multi-center study. Five centers met these criteria and agreed to participate (Torino, Verona, Ancona, Roma, and Napoli). Inclusion criteria for participants were a diagnosis of T1D for over six months, between the ages of 2-17 years, using Dexcom G6 CGM System (Dexcom, Inc., San Diego, CA, USA) for over six months, having an active connection using Clarity ${ }^{\circledR}$ software (Dexcom international Switzerland, Horw), an HbA1c $\leq 10 \%$ $(86 \mathrm{mmol} / \mathrm{L})$ during the three months prior to recruitment, and parents available to collect and record nutritional information for three days. To avoid potential discrepancies due to different accuracies of various CGMs, only patients using a Dexcom G6 were recruited. Exclusion criteria were unwillingness to use the CGM, use of a CGM other than Dexcom G6, unwillingness to share glucose data with the center, unavailability or inability of the parents to collect nutritional data, diagnosis of celiac disease, use of predictive low glucose management or CLC system, and $\mathrm{HbA} 1 \mathrm{c}>10 \%$ before the study. This study was approved by the Independent Ethics Committees of all five participating centers.

\subsection{Study Procedures}

From January 2019 to January 2020, before the start of the SARS-CoV-2 outbreak in Italy, all participants meeting the eligibility criteria were invited to participate in the study during their scheduled annual visit. All participating parents and adolescents over the age of 14 provided written informed consent. At the annual visit, a qualified researcher collected demographic information and clinical data on diabetes management. Additionally, parents had previously given written permission to connect their child's Dexcom G6 blood glucose sensor (Dexcom international Switzerland, Horw) to the cloud connected to the pediatric diabetes centers. During the annual visit, this link was verified. Finally, a blood sample was added to the clinical examination to study T1D complications. 


\subsection{Nutritional Assessment}

Dietary intake was assessed using a three-day weighed dietary record. A trained dietitian in each center advised families that the main purpose of the study was to analyze TIR with usual daily intake of food, so participants were advised to maintain all their current daily eating habits during the study. A kitchen scale (Soehnle Digital) and a food diary were provided. Parents of patients included in the study were carefully instructed on how to collect data and were provided with instructions on how to evaluate foods and record data using the validated DONALD study's three-day weighed food diary [14] Parents' ability to measure nutrient intake was verified by the dietitian by way of a practical test before the study. They were asked to collect data in the food diary for three days (Sunday, Monday, and Tuesday) of the two weeks following recruitment. Through the food diary, they provided information about type and brand names of food items, time and location of eating, and recipes. For commercial food items, the packages or the food labels were collected and the product information was added to the dietary record using Winfood ${ }^{\circledR}$ software (Medimatica, Teramo, Italy). Semi-quantitative recording was allowed when weighing was not possible, e.g., eating meals or snacks away from home.

\subsection{Variables}

Clinical and demographic characteristics included date of birth and date of diabetes diagnosis, gender, weight, height, number of episodes of severe hypoglycemia or ketoacidosis in the last 12 months, glycated hemoglobin (HbA1c), lipid profile (total cholesterol, HDL cholesterol, LDL cholesterol, triglycerides), weekly hours of physical activity, type of insulin therapy (MDI or CSII), average total daily insulin dose during the preceding week, and the use of a carbohydrate counting system. All centers used the same analytical laboratory methods. Total cholesterol, HDL cholesterol, and triglycerides were measured in stored plasma samples by an enzymatic method using a Beckman Coulter Olympus AU 480 (Beckman Coulter, Brea, CA, USA), and LDL was calculated using the Friedewald equation, which includes total cholesterol, HDL cholesterol, and triglycerides. HbA1c was measured with the DCA Vantage ${ }^{\circledR}$ Analyzer.

Continuous glucose monitor-based glucose metrics (TIR, percentage of time $<54 \mathrm{mg} / \mathrm{dL}$, $54-70 \mathrm{mg} / \mathrm{dL} 180-250 \mathrm{mg} / \mathrm{dL}$, and $>250 \mathrm{mg} / \mathrm{dL}$, coefficient of variation, percentage of time CGM was active) were collected during the 15 days following the recruitment visit in which the participants' food diaries were collected.

Food diaries were coded and quantified by the same dietitian (MM) for all centers. The dietary intake was assessed by calculating the total daily kilocalorie intake and the percentages of sugars, total carbohydrates, saturated fatty acids, monounsaturated fatty acids, polyunsaturated fatty acids, and protein intake in diets. The amount of daily cholesterol (g/day) and daily fiber (g/day) were also assessed as part of the dietary intake.

\subsection{Statistical Analysis}

The Shapiro-Wilk test results showed that quantitative variables were not normally distributed, so a non-parametric approach was used for the analysis.

The characteristics of the study sample were evaluated according to the percentage of time with glucose between 70 and $180 \mathrm{mg} / \mathrm{dL}$ (TIR $\leq 70 \%$ and $>70 \%$ ). Quantitative variables were summarized using medians and interquartile ranges and qualitative variables as absolute and percentage frequencies. Group comparisons between those with TIRs $>70 \%$ and those with TIRs $\leq 70 \%$ were performed using the Wilcoxon-Mann-Whitney test in the case of quantitative variables and the chi-squared test or Fisher's exact test (when expected frequencies were $<5$ ) for qualitative variables.

Multiple correspondence analysis (MCA), an exploratory statistical technique, was used to detect all the characteristics common to participants with T1D who had a percentage TIR $>70 \%$. Metabolic control $\left(\mathrm{HbA}_{1 \mathrm{c}}\right.$ grouped as either $<7 \%$ and $\left.\geq 7 \%[53 \mathrm{mmol} / \mathrm{mol}]\right)$, insulin delivery system (CSII or MDI), use of the carbohydrate counting system (yes or no), percentage of total carbohydrate (CHO: $<40,40-44,45-50,>50)$, protein (P: $<15,15-20$, 
$>20)$, and fat $(<35, \geq 35)$, and TIR levels were analyzed simultaneously. MCA organizes the modalities of categorical variables into a multiple contingency table to calculate row and column frequencies. The frequencies are then projected onto a Cartesian plane to obtain a graphical representation of the associations between the variables. The modalities that are close to each other are those shared by the same patients, and the groupings of the modalities allow the interpretation of associations between the variables. The overall variability explained by the MCA model is indicated by the inertia, assuming a value equal to $100 \%$ if the model is able to explain all the variability.

Multiple logistic regression analysis was used to estimate the independent effect of patients' clinical and nutritional characteristics on the probability of having a TIR $>70 \%$. All estimates were obtained calculating $95 \%$ confidence intervals (CIs). The likelihood ratio (LR) test and Hosmer-Lemeshow test were used to select the most parsimonious model and to evaluate the model's goodness of fit.

A probability $<0.05$ was used to assess statistical significance, and all statistical analyses were performed using $\mathrm{R}$ version 4.0.4.

\section{Results}

A total of 197 children and adolescents were enrolled in this study. The overall median TIR was $60 \%$ (IQR $47-71 \%$ ). The clinical characteristics and CGM-based glucose metrics of the participants according to the TIR cut-off are shown in Table 1. Fifty-five participants [27.9\% (95\% CI 21.8-34.7)] reported a TIR > 70\%, with a median score of 77 (IQR 70-82).

Table 1. Clinical characteristics according to percentage of time in range.

\begin{tabular}{|c|c|c|c|c|}
\hline & \multirow{2}{*}{$\begin{array}{c}\text { Total } \\
(n=197)\end{array}$} & \multicolumn{2}{|c|}{$\%$ Time $70-180 \mathrm{mg} / \mathrm{dL}$} & \multirow[b]{2}{*}{$p$-Value } \\
\hline & & $\leq 70(n=142)$ & $>70(n=55)$ & \\
\hline \multicolumn{5}{|l|}{ Clinics } \\
\hline Sex, F & $92(46.7)$ & $64(45.1)$ & $28(50.9)$ & 0.564 \\
\hline Age, years & $11.6(8.6 ; 14.3)$ & $11.4(8.2 ; 14)$ & $11.9(9.5 ; 15)$ & 0.069 \\
\hline Pubertal stage, Tanner $2-5$ & $119(60.4)$ & $82(57.7)$ & $37(67.3)$ & 0.287 \\
\hline Insulin delivery System, CSII & $93(47.2)$ & $69(48.6)$ & $24(43.6)$ & 0.641 \\
\hline Diabetes duration, years & $3.7(2 ; 6.8)$ & $4.7(2.5 ; 7.3)$ & $2.1(1.1 ; 5.5)$ & 0.001 \\
\hline BMI SDS & $0.2(-0.5 ; 0.7)$ & $0.2(-0.4 ; 0.7)$ & $-0.1(-0.6 ; 0.7)$ & 0.469 \\
\hline Physical activity, hours/w & $3(2 ; 4)$ & $3(1 ; 4)$ & $3(2 ; 5)$ & 0.150 \\
\hline $\mathrm{HbA} 1 \mathrm{c}, \%$ & $7.2(6.5 ; 7.7)$ & $7.4(6.9 ; 7.9)$ & $6.4(6 ; 6.7)$ & $<0.001$ \\
\hline Total cholesterol, mg/dL & $155(140 ; 175)$ & $157(142 ; 176)$ & $153(135 ; 174)$ & 0.304 \\
\hline Cholesterol HDL, mg/dL & $59(49 ; 71)$ & $58(49 ; 71.2)$ & $60(48 ; 70)$ & 0.723 \\
\hline Cholesterol LDL, mg/dL & $88.4(73 ; 103)$ & $89(73.3 ; 102.6)$ & $84(72.5 ; 103)$ & 0.666 \\
\hline Triglycerides, mg/dL & $53(43 ; 67)$ & $53.5(43 ; 67.2)$ & $51(43.5 ; 63)$ & 0.771 \\
\hline Carb counting system, Yes & $92(46.7)$ & $60(42.3)$ & $32(58.2)$ & 0.064 \\
\hline \multicolumn{5}{|l|}{ CGM-based glucose metrics } \\
\hline$\%$ time $<54 \mathrm{mg} / \mathrm{dL}$ & $0.2(0 ; 0.9)$ & $0.2(0 ; 0.9)$ & $0.3(0 ; 0.9)$ & 0.552 \\
\hline$\%$ time $54-70 \mathrm{mg} / \mathrm{dL}$ & $2(0.5 ; 4.5)$ & $1.5(0.4 ; 4)$ & $3(1.4 ; 5.2)$ & 0.010 \\
\hline$\%$ time $180-250 \mathrm{mg} / \mathrm{dL}$ & $37(24 ; 50)$ & $43(35 ; 56)$ & $17.9(12.2 ; 23)$ & $<0.001$ \\
\hline$\%$ time $>250 \mathrm{mg} / \mathrm{dL}$ & $10(4 ; 20)$ & $14.5(9.1 ; 25.4)$ & $2.2(0.7 ; 3.8)$ & $<0.001$ \\
\hline$\% \mathrm{CV}$ & $36(32 ; 41)$ & $37.6(32.9 ; 41)$ & $33.6(30.2 ; 36.9)$ & $<0.001$ \\
\hline$\%$ time CGM active & $95(89 ; 98)$ & $93.1(85.7 ; 97.6)$ & $97.3(93.8 ; 98.7)$ & $<0.001$ \\
\hline
\end{tabular}

Values are presented as median (IQR) or $n(\%) ; p$-values refer to Wilcoxon rank-sum test or chi-square test.

Participants with TIR $>70 \%$ had significantly shorter disease duration and lower $\mathrm{HbA1c}$ levels. No significant differences were found between the two groups in terms of other clinical and lipid characteristics. Moreover, a TIR $>70 \%$ was significantly associated with lower percentage of time in hyperglycemia (\% time $180-250 \mathrm{mg} / \mathrm{dL}$ and $>250$ ), higher percentage of time in hypoglycemia (\% time $54-70 \mathrm{mg} / \mathrm{dL}$ ), lower $\% \mathrm{CV}$, and higher percentage of time with the CGM active. 
The nutritional profiles of participants according to TIR levels were also evaluated (Table 2). Patients with a TIR $>70 \%$ consumed a significantly higher percentage of protein and fiber per day. No significant differences were found between the two groups with respect to the consumption of other nutrients. Overall, 17 participants $(8.6 \%)$ met all the three macronutrient ISPAD goals [15], with no significant difference between patients with a TIR $>70 \%$ or TIR $\leq 70 \%$. Participants with a TIR $>70 \%$ more frequently consumed a carbohydrate percentage between $40 \%$ and $44 \%$ and a protein percentage of $>20 \%$. A protein percentage $<15 \%$ was significantly more frequent in participants with a TIR $\leq 70 \%$. No significant differences were found between the two groups when considering overall fat, saturated fat, and fiber targets separately.

Table 2. Subjects' nutritional profiles according to percentage of time in range.

\begin{tabular}{|c|c|c|c|c|}
\hline & \multirow{2}{*}{$\begin{array}{c}\text { Total } \\
(n=197)\end{array}$} & \multicolumn{2}{|c|}{ \% Time 70-180 mg/dL } & \multirow[b]{2}{*}{$p$-Value } \\
\hline & & $\leq 70(n=142)$ & $>70(n=55)$ & \\
\hline \multicolumn{5}{|l|}{ Nutrients [median (IQR)] } \\
\hline Kcal/day & $1668(1495-1943)$ & 1675 (1496-1940) & 1615 (1488-1943) & 0.689 \\
\hline Protein & $16.9(14.4 ; 19)$ & $16.3(14.1 ; 18.3)$ & $17.6(15.8 ; 19.4)$ & 0.015 \\
\hline Carbohydrate & $45.9(42.3 ; 49.1)$ & $46.2(42.4 ; 49.1)$ & $43.4(41.5 ; 48.2)$ & 0.098 \\
\hline Fat & $37.3(33.3 ; 41.0)$ & $37.6(33.4 ; 37.2)$ & $37.2(33.2 ; 41.2)$ & 0.750 \\
\hline SFA & $9.6(7.8 ; 10.9)$ & $9.5(7.8 ; 10.7)$ & $9.1(7.6 ; 10.9)$ & 0.753 \\
\hline PUFA & $10.3(7 ; 14.9)$ & $11.2(7 ; 15.1)$ & $9.2(6.3 ; 13.3)$ & 0.196 \\
\hline MUFA & $16.4(13.8 ; 19.4)$ & $16.1(13.7 ; 19.1)$ & $17.4(15 ; 20.3)$ & 0.211 \\
\hline Sugar & $11.1(8.2 ; 13.6)$ & $11.2(8.2 ; 13.7)$ & $11.1(8.6 ; 12.7)$ & 0.741 \\
\hline Fiber (g/day) & $13.1(10.2 ; 16.3)$ & $12.8(9.6 ; 15.7)$ & $14.4(11.7 ; 17.1)$ & 0.031 \\
\hline \multicolumn{5}{|l|}{ ISPAD nutritional goals, $n(\%)$} \\
\hline All macronutrient goals & $17(8.6)$ & $11(7.7)$ & $6(10.9)$ & 0.572 \\
\hline $\mathrm{CHO}<40 \%$ & $31(15.7)$ & $24(16.9)$ & $7(12.7)$ & 0.615 \\
\hline $40-44 \%$ & $53(26.9)$ & $29(20.4)$ & $24(43.6)$ & 0.002 \\
\hline $45-50 \%$ & $76(38.6)$ & $60(42.3)$ & $16(29.1)$ & 0.124 \\
\hline$>50 \%$ & $37(18.8)$ & $29(20.4)$ & $8(14.5)$ & 0.457 \\
\hline FAT $<35 \%$ & $61(31.0)$ & $41(28.9)$ & $20(36.4)$ & 0.309 \\
\hline Saturated fat $<10 \%$ & $110(55.8)$ & $78(54.9)$ & $32(58.20)$ & 0.750 \\
\hline Protein $<15 \%$ & $60(30.5)$ & $50(35.2)$ & $10(18.2)$ & 0.031 \\
\hline $15-20 \%$ & $113(57.4)$ & $79(55.6)$ & $34(61.8)$ & 0.531 \\
\hline$>20 \%$ & $24(12.2)$ & $13(9.2)$ & $11(20.0)$ & 0.031 \\
\hline Fiber $\geq$ age (years) +5 & $54(27.4)$ & $37(26.1)$ & $17(30.9)$ & 0.483 \\
\hline
\end{tabular}

All macronutrient goals refer to meeting goals for carbohydrate, fat, and protein simultaneously. Values are presented as median (IQR) or $\mathrm{n}(\%)$; p-values refer to the Wilcoxon rank-sum test or chi-squared test. Abbreviations: P: protein; SFA: saturated fatty acid; PUFA: polyunsaturated fatty acids; MUFA: monounsaturated fatty acids.

Figure 1 shows the results of the MCA. The two identified dimensions explained 51\% of the total variability. It was possible to identify four groups, as follows:

(1) The first group in the second quadrant (II) contained participants with a TIR $>70 \%$ with optimal metabolic control. They consumed a protein percentage $>20 \%$ and carbohydrate between $40 \%$ and $44 \%$.

(2) The opposite quadrant (IV) contained participants that did not have good metabolic control $\left(\mathrm{HbA}_{1 \mathrm{c}} \geq 7 \%[53 \mathrm{mmol} / \mathrm{mol}]\right.$ and TIR $\left.\leq 70 \%\right)$. They consumed a low protein percentage $(<15 \%)$ and a carbohydrate percentage between $45 \%$ and $50 \%$.

(3) The first quadrant (I) contained participants using the carbohydrate counting system and CSII, and they consumed $>50 \%$ carbohydrate and a low percentage of fat $(<35 \%)$.

(4) The third quadrant (III) contained participants characterized by a protein percentage between $15 \%$ and $20 \%$, a high percentage of fat $(\geq 35 \%)$, and a low percentage of carbohydrate $(<40 \%)$, treated with MDI, and not using the carbohydrate counting system.

Quadrants II and IV identified a factorial plane in which patients consuming less carbohydrate and more protein with respect to ISPAD guidelines and at metabolic target were 
opposite patients characterized by poor metabolic control consuming the recommended percentage of carbohydrate as per ISPAD guidelines.

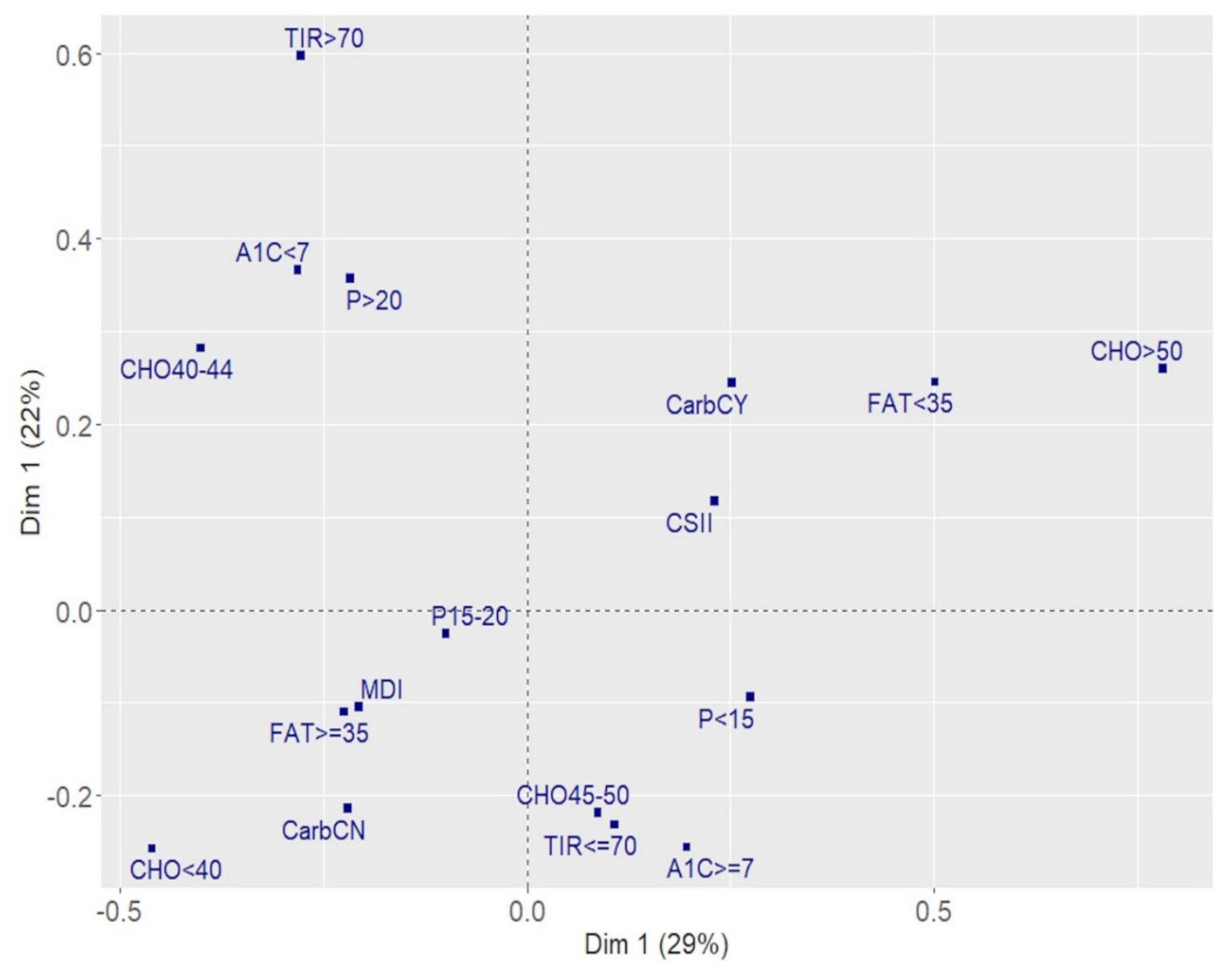

Figure 1. Associations between nutrients and metabolic control. Results of the multiple correspondence analysis. A1c $>7$ : $\mathrm{HbA} 1 \mathrm{c}>7 \%$ (53 mmol/mol); A1c < 7: HbA1c $\leq 7 \%$ (53 mmol/mol); CSII: insulin pump; MDI: multiple daily injection; CarbCN: no use of a carbohydrate counting system; CarbCY: use of a carbohydrate counting system; $\mathrm{CHO}<40: \%$ of total carbohydrate less than 40\%; CHO40-44: between 40\% and 44\%; $\mathrm{CHO} 45-50$ : between $45 \%$ and 50\%; $\mathrm{CHO}>50$ : greater than $50 \%$; FAT < 35: $\%$ of fat $<35 \%$; FAT $\geq 35: \%$ of fat $\geq 35 \%$; P < 15: \% of protein less than $15 \%$; P15-20: between $15 \%$ and $20 \%$; P > 20: greater than 20\%; TIR > 70: \% time 70-180 mg/dL more than 70\%; TIR $\leq 70: \%$ time $70-180 \mathrm{mg} / \mathrm{dL}$ up to $70 \%$.

Table 2 shows the results of multiple logistic regression analysis adjusting for age, diabetes duration, insulin dose (units $/ \mathrm{kg} /$ day), and $\mathrm{kcal} / \mathrm{kg} /$ day intake. The probability of having a TIR $>70 \%$ was significantly higher with a percentage carbohydrate consumption between $40 \%$ and $44 \%$ compared with a percentage of $45-50 \%$ and with the use of a carbohydrate counting system. The longer the disease duration, the lower the probability of having a TIR > 70\%; the higher the age, the lower the units of insulin dose $/ \mathrm{kg}$; and the lower the energy intake $(\mathrm{kcal} / \mathrm{kg})$ per day, the higher the probability of having a TIR $>70 \%$.

\section{Discussion}

To our best knowledge, this is the first study reporting associations between TIR $\geq 70 \%$ and macronutrient intake in children and adolescents with T1D. Our results showed that participants consuming a diet with a carbohydrate percentage of $40-44 \%$ were more frequently at target for TIR and HbA1c. Moreover, the probability of being at target was significantly higher when compared to those with carbohydrate intake of $45-50 \%$ (Table 3). 
Table 3. Factors associated with target TIR (>70\%).

\begin{tabular}{cccc}
\hline Variable & OR & $\mathbf{9 0 \% C I}$ & $p$-Value \\
\hline Total CHO (<40\% vs. 45-50\%) & 0.2 & $0.04-0.81$ & 0.033 \\
Total CHO (40-44\% vs. 45-50\%) & 2.56 & $1.05-6.37$ & 0.039 \\
Total CHO (>50\% vs. 45-50\%) & 0.73 & $0.23-2.19$ & 0.577 \\
P (15-20\% vs. <15\%) & 1.78 & $0.73-4.58$ & 0.213 \\
P (>20\% vs. <15\%) & 2.3 & $0.54-9.81$ & 0.256 \\
CHO counting (yes vs. no) & 2.29 & $1.05-5.12$ & 0.039 \\
Diabetes duration (years) & 0.76 & $0.65-0.88$ & $<0.001$ \\
Age (years) & 1.31 & $1.14-1.53$ & $<0.001$ \\
Insulin dose (units/kg/day) & 0.02 & $0.00-0.15$ & $<0.001$ \\
BMI SDS & 0.79 & $0.51-1.23$ & 0.299 \\
Kcal/day & 0.99 & $0.98-1.00$ & 0.664 \\
\hline
\end{tabular}

Results from logistic regression analysis; LR test: $\chi^{2}$ with $11 \mathrm{df}, \chi^{2}=60.14, p<0.001$; Hosmer and Lemeshow goodness of fit test: $\chi^{2}$ with $8 \mathrm{df}, \chi^{2}=7.47, p=0.487$.

The median TIR of the entire cohort did not reach the established upper target of greater than $70 \%$, as suggested for patients with T1D [15]; however, it reached the minimum criterion of TIR at $60 \%$ if the $\mathrm{HbA} 1 \mathrm{c}$ goal is $7.5 \%$, as indicated for young people under the age of 25 years.

Our results showed that the probability of reaching a TIR $>70 \%$ increased with age. This is consistent with American Diabetes Association recommendations indicating that glycemic goals may need to be modified to consider the fact that younger children may have a form of "hypoglycemic unawareness", in that they lack the cognitive capacity to recognize and respond to hypoglycemic symptoms and may be at greater risk of sequelae from hypoglycemia. In addition, children below the age of 5 years may be at risk of permanent cognitive impairment after episodes of severe hypoglycemia [16]. Therefore, more effort is needed to improve TIR in younger children by increasing parents' diabetes education, refining insulin treatment [17], or using more advanced technological systems such as closed-loop control, which reduces the occurrence of severe hypoglycemia [18,19].

It is also noteworthy that participants' body weights and lipid profiles were within the normal range for age and gender. The lipid profile of our series was comparable to a previous report of Italian children with type 1 diabetes [6] showing a mean LDL of $92 \mathrm{mg} / \mathrm{dL}$ and HDL of $58.5 \mathrm{mg} / \mathrm{dL}$; in our study, no difference was detected in LDL and HDL values between participants achieving a TIR $>70 \%$ and those who did not.

Importantly, only $8.6 \%$ of all participants met current ISPAD macronutrient recommendations for all components, with no significant differences between those who achieved a TIR $>70 \%$ and those who did not. On the other hand, only $38.6 \%$ and $31.0 \%$ of all participants consumed carbohydrates and fats according to ISPAD guidelines, respectively.

To date, the association between macronutrient distribution and glycemic control has mainly focused on $\mathrm{HbA1c}[4,6-8,20]$, which is the best known marker of complication risk and commonly assessed quarterly as suggested by diabetes clinical practice guidelines. On the other hand, the use of $\mathrm{HbA1c}$ alone for assessing glycemic control can be misleading [20], as the same $\mathrm{HbA1c}$ value can be associated with good, fair, or even poor glycemic control as judged by the different potential mean glucose levels calculated by the CGM. In addition, observational studies analyzing macronutrient intake have usually been based on a threeday food diary record, while HbA1c reflects blood glucose concentrations over 3-4 months. As macronutrient intake may vary over the 3-4-month period, the association between $\mathrm{HbA} 1 \mathrm{c}$ and macronutrient intake can be unreliable.

CGM-based glycemic metrics allow for the relationship between nutritional intake and glycemic pattern to be measured over a short period of time such as two weeks or even less. Hence, CGM metrics may be more helpful in diabetes management with regards to the type and quantity of macronutrients consumed.

While several nutritional factors may influence metabolic control, carbohydrate intake is one of the primary determinants of postprandial glucose levels. Results from the Diabetes 
Complication and Control Trial (DCCT) showed that participants randomly assigned to intensive therapy with a dietary intake higher in fat and saturated fat and lower in carbohydrate had worse glycemic control [20]. On the other hand, results from a longitudinal study reported that the higher the carbohydrate intake, the more the HbA1c increased [21]. The recommended carbohydrate intake in the 2014 ISPAD guidelines [22] of 50-55\% of total energy intake was reduced in the 2018 ISPAD guidelines [1] to $45-50 \%$. To assess the percentage of carbohydrate intake associated with the best metabolic control as measured by TIR, we considered four classes. Using the $45-50 \%$ class as a reference, we found that the probability of having an TIR $>70 \%$ increased more than 2.5 times if carbohydrate intake was between $40-44 \%$. Moreover, patients consuming a carbohydrate percentage of $40-44 \%$ more frequently had an $\mathrm{HbA} 1 \mathrm{c}<7 \%$ (Figure 1 ). In addition, the carbohydrate counting system was associated with a higher probability of TIR $>70 \%$ (Table 3 ) and it was used more frequently by participants consuming a carbohydrate percentage $>50 \%$ (Figure 1). The use of the carbohydrate counting system therefore represents a key strategy for improving metabolic control and reaching the target TIR. Its use should continue to be recommended in the daily management of diabetes.

In our analysis, the overall sugar intake among all participants was lower than that reported in previous studies $[6,10,23]$, with no difference between participants who achieved or did not achieve the target TIR. However, it should be noted that, due to the study design, the amount of sugar used to correct hypoglycemia could not be analyzed. Participants achieving a TIR $>70 \%$ consumed more fiber per day (Table 2); however, the impact of this nutrient was not significant when considered in the multiple analysis.

Previous studies would have used traditional blood glucose monitoring and not a real time CGM device. A recent study using data from the DCCT showed that decreased TIR was strongly associated with the risk of microvascular complications and, although $\mathrm{HbA1c}$ remains a key outcome measure, TIR can add value as an outcome measure [23]. As a result of the widespread use of CGM and increased accuracy of results, we can now more widely use TIR as a measure in clinical practice. The results of our study need confirmation in a larger sample and in populations that have different dietary intakes. If confirmed, these data could have implications in clinical practice, because small changes in diet could increase the percentage of TIR in children and adolescents with T1D.

Our study has a few limitations. The cross-sectional design did not allow us to prospectively assess an association between macronutrients and metabolic control. Also, the inclusion of patients using an insulin pump system based on different algorithms did not allow for an analysis of the effect of technologies on the probability of achieving target TIR. Evaluating the effect of the macronutrient composition in patients using the same algorithm remains an area of investigation. Nevertheless, this study had many strengths including a multi-center design, data collected from many participants with well-characterized T1D, and use of the same analytical laboratory methods and food consumption measure. There was consistency in the training for parents, and the macronutrient intake calculations were conducted by the same dietitian for all the centers involved. Moreover, parents' ability to measure nutrient intake was tested before the study, which helped to eliminate any potential errors in assessing food consumption. Finally, there were no potential conflicts of interest with Dexcom regarding the use of this glucose sensor that affected the study protocol or results.

\section{Conclusions}

This study shows that a small reduction in the percentage of carbohydrate intake to $40-44 \%$, compared to the current ISPAD guidelines which indicate $45-50 \%$, improves the percentage of TIR. This allows children and adolescents with type 1 diabetes to achieve the goal of a TIR greater than $70 \%$. Additionally, both a lower and higher percentage of carbohydrate intake than the ISPAD guidelines appears to reduce the probability of reaching the target TIR by $70 \%$. 
It should be noted that increasing the TIR requires a multifactorial approach that takes into account modifiable factors such as macronutrient intake, type of treatment, insulin titration, physical activity as well as non-modifiable factors such as age, diabetes duration, and experienced hypoglycemia unawareness.

These findings require validation in other populations before they can be incorporated into clinical practice guidelines and remain an area of future research. Further studies are necessary to investigate TIR and macronutrients in children from different socioeconomic and ethnic backgrounds.

Author Contributions: V.C. had full access to the data used in this article and is the guarantor of this study. V.C., M.M. (Monica Marino), M.M. (Marco Marigliano), C.M., A.Z., I.R., S.G., R.S., A.L., S.R., A.I., D.I., A.E.S., R.B. and R.G. contributed to the study design, data collection, and drafting and gave the final approval for this version to be published. R.B. contributed to the discussion and revision of the manuscript. R.G. performed and was responsible for the integrity of the data and accuracy of the data analysis. M.M. (Monica Marino) was responsible for the calculation of macronutrient components of food diaries. All authors have read and agreed to the published version of the manuscript.

Funding: No funding was received for the preparation of this manuscript.

Institutional Review Board Statement: In this section, please add the Institutional Review Board Statement and approval number for studies involving humans or animals. Please note that the Editorial Office might ask you for further information. Please add "The study was conducted according to the guidelines of the Declaration of Helsinki, and approved by the Institutional Review Board (or Ethics Committee) of Comitato Etico Regionale delle Marche(protocol code DT1AAVG2018, 27 September 2018, $\mathrm{n}^{\circ} 249$ ). You might also choose to exclude this statement if the study did not involve humans or animals.

Informed Consent Statement: Informed consent was obtained from all subjects involved in the study.

Data Availability Statement: Data is contained within the article.

Acknowledgments: The authors thank the young people and parents who kindly took part in this study.

Conflicts of Interest: The authors declare no conflict of interest.

\section{References}

1. Smart, C.E.; Annan, F.; Higgins, L.A.; Jelleryd, E.; Lopez, M.; Acerini, C.L. ISPAD Clinical Practice Consensus Guidelines 2018: Nutritional Management in Children and Adolescents with Diabetes. Pediatr. Diabetes 2018, 19, 136-154. [CrossRef]

2. $\quad$ Evert, A.B.; Boucher, J.L.; Cypress, M.; Dunbar, S.A.; Franz, M.J.; Mayer-Davis, E.J.; Neumiller, J.J.; Nwankwo, R.; Verdi, C.L.; Urbanski, P.; et al. Nutrition Therapy Recommendations for the Management of Adults with Diabetes. Diabetes Care 2014, 37, 120-143. [CrossRef]

3. Rovner, A.J.; Nansel, T.R. Are Children with Type 1 Diabetes Consuming a Healthful Diet?: A Review of the Current Evidence and Strategies for Dietary Change. Diabetes Educ. 2009, 35, 97-107. [CrossRef]

4. Seckold, R.; Howley, P.; King, B.R.; Bell, K.; Smith, A.; Smart, C.E. Dietary Intake and Eating Patterns of Young Children with Type 1 Diabetes Achieving Glycemic Targets. BMJ Open Diabetes Res. Care 2019, 7, e000663. [CrossRef] [PubMed]

5. Mayer-Davis, E.J.; Nichols, M.; Liese, A.D.; Bell, R.A.; Dabelea, D.M.; Johansen, J.M.; Pihoker, C.; Rodriguez, B.L.; Thomas, J.; Williams, D. Dietary Intake among Youth with Diabetes: The SEARCH for Diabetes in Youth Study. J. Am. Diet. Assoc. 2006, 106, 689-697. [CrossRef]

6. Maffeis, C.; Morandi, A.; Ventura, E.; Sabbion, A.; Contreas, G.; Tomasselli, F.; Tommasi, M.; Fasan, I.; Costantini, S.; Pinelli, L. Diet, Physical, and Biochemical Characteristics of Children and Adolescents with Type 1 Diabetes: Relationship between Dietary Fat and Glucose Control. Pediatr. Diabetes 2012, 13, 137-146. [CrossRef]

7. Øverby, N.C.; Margeirsdottir, H.D.; Brunborg, C.; Andersen, L.F.; Dahl-Jørgensen, K. The Influence of Dietary Intake and Meal Pattern on Blood Glucose Control in Children and Adolescents Using Intensive Insulin Treatment. Diabetologia 2007, 50, $2044-2051$. [CrossRef] [PubMed]

8. Katz, M.L.; Mehta, S.; Nansel, T.; Quinn, H.; Lipsky, L.M.; Laffel, L.M.B. Associations of Nutrient Intake with Glycemic Control in Youth with Type 1 Diabetes: Differences by Insulin Regimen. Diabetes Technol. Ther. 2014, 16, 512-518. [CrossRef] [PubMed]

9. Nansel, T.R.; Lipsky, L.M.; Liu, A. Greater Diet Quality Is Associated with More Optimal Glycemic Control in a Longitudinal Study of Youth with Type 1 Diabetes. Am. J. Clin. Nutr. 2016, 104, 81-87. [CrossRef]

10. Beck, R.W.; Bergenstal, R.M.; Riddlesworth, T.D.; Kollman, C.; Li, Z.; Brown, A.S.; Close, K.L. Validation of Time in Range as an Outcome Measure for Diabetes Clinical Trials. Diabetes Care 2019, 42, 400-405. [CrossRef] 
11. Advani, A. Positioning Time in Range in Diabetes Management. Diabetologia 2020, 63, 242-252. [CrossRef] [PubMed]

12. Cherubini, V.; Bonfanti, R.; Casertano, A.; De Nitto, E.; Iannilli, A.; Lombardo, F.; Maltoni, G.; Marigliano, M.; Bassi, M.; Minuto, N.; et al. Time in Range in Children with Type 1 Diabetes Using Treatment Strategies Based on Nonautomated Insulin Delivery Systems in the Real World. Diabetes Technol. Ther. 2020, 22, 509-515. [CrossRef] [PubMed]

13. Schoelwer, M.J.; Kanapka, L.G.; Wadwa, R.P.; Breton, M.D.; Ruedy, K.J.; Ekhlaspour, L.; Forlenza, G.P.; Cobry, E.C.; Messer, L.H.; Cengiz, E.; et al. Predictors of Time-in-Range (70-180 mg/dL) Achieved Using a Closed-Loop Control System. Diabetes Technol. Ther. 2021, 23, 475-481. [CrossRef] [PubMed]

14. Kroke, A.; Manz, F.; Kersting, M.; Remer, T.; Sichert-Hellert, W.; Alexy, U.; Lentze, M.J. The DONALD Study: History, Current Status and Future Perspectives. Eur. J. Nutr. 2004, 43, 45-54. [CrossRef]

15. Battelino, T.; Danne, T.; Bergenstal, R.M.; Amiel, S.A.; Beck, R.; Biester, T.; Bosi, E.; Buckingham, B.A.; Cefalu, W.T.; Close, K.L.; et al. Clinical Targets for Continuous Glucose Monitoring Data Interpretation: Recommendations From the International Consensus on Time in Range. Diabetes Care 2019, 42, 1593-1603. [CrossRef]

16. Statements, P. Standards of Medical Care in Diabetes-2012. Diabetes Care 2012, 35, S11-S63. [CrossRef]

17. Danne, T.; Phillip, M.; Buckingham, B.A.; Jarosz-Chobot, P.; Saboo, B.; Urakami, T.; Battelino, T.; Hanas, R.; Codner, E. ISPAD Clinical Practice Consensus Guidelines 2018: Insulin Treatment in Children and Adolescents with Diabetes. Pediatr. Diabetes 2018, 19, 115-135. [CrossRef]

18. Breton, M.D.; Kanapka, L.G.; Beck, R.W.; Ekhlaspour, L.; Forlenza, G.P.; Cengiz, E.; Schoelwer, M.; Ruedy, K.J.; Jost, E.; Carria, L.; et al. A Randomized Trial of Closed-Loop Control in Children with Type 1 Diabetes. N. Engl. J. Med. 2020, 383, 836-845. [CrossRef] [PubMed]

19. Beck, R.W.; Connor, C.G.; Mullen, D.M.; Wesley, D.M.; Bergenstal, R.M. The Fallacy of Average: How Using Hba1c Alone to Assess Glycemic Control Can Be Misleading. Diabetes Care 2017, 40, 994-999. [CrossRef] [PubMed]

20. Delahanty, L.M.; Nathan, D.M.; Lachin, J.M.; Hu, F.B.; Cleary, P.A.; Ziegler, G.K.; Wylie-Rosett, J.; Wexler, D.J. Association of Diet with Glycated Hemoglobin during Intensive Treatment of Type 1 Diabetes in the Diabetes Control and Complications Trial 1-3 for the Diabetes Control and Complications Trial/Epidemiology of Diabetes Interventions and Complications. Am. J. Clin. Nutr. 2009, 89, 518-524. [CrossRef]

21. Lamichhane, A.P.; Crandell, J.L.; Jaacks, L.M.; Couch, S.C.; Lawrence, J.M.; Mayer-Davis, E.J. Longitudinal Associations of Nutritional Factors with Glycated Hemoglobin in Youth with Type 1 Diabetes: The SEARCH Nutrition Ancillary Study. Am. J. Clin. Nutr. 2015, 101, 1278-1285. [CrossRef] [PubMed]

22. Smart, C.E.; Annan, F.; Bruno, L.P.C.; Higgins, L.A.; Acerini, C.L. Nutritional Management in Children and Adolescents with Diabetes. Pediatr. Diabetes 2014, 15, 135-153. [CrossRef] [PubMed]

23. Maffeis, C.; Tomasselli, F.; Tommasi, M.; Bresadola, I.; Trandev, T.; Fornari, E.; Marigliano, M.; Morandi, A.; Olivieri, F.; Piona, C. Nutrition Habits of Children and Adolescents with Type 1 Diabetes Changed in a 10 Years Span. Pediatr. Diabetes 2020, 21, 960-968. [CrossRef] [PubMed] 